\title{
Conocimiento y aprendizaje desde la asignatura de Rol e Identidad del Trabajo Social.
}

\author{
Knowledge and learning in the course 'Role and Identity' in Social Work.
}

\section{Resumen}

En este artículo se plantean las bases conceptuales y metodológicas de la asignatura de Rol e Identidad del Grado de Trabajo Social de la Universidad de Barcelona. El objetivo principal del artículo es realizar un paseo reflexivo conceptual, en torno a la propuesta pedagógica y teórica de la asignatura, que pueda ser de interés tanto para los y las estudiantes de Trabajo Social como para los y las profesionales. Por ello, el artículo está configurado de forma ensayística, para favorecer la argumentación y la exposición teórico-reflexiva derivada de las experiencias, conocimientos y aprendizajes de los y las docentes que han formado parte de esta asignatura.

Este recorrido teórico de la asignatura se aborda desde dos dimensiones: la dimensión ontológica del contenido del plan docente y la dimensión epistemológica de la metodología educativa. De este modo, se refleja la necesidad de los y las estudiantes de Trabajo Social de compenetrarse con la complejidad para la comprensión de su realidad personal y profesional, y sitúa la narrativa (auto)biográfica como la estrategia que permite al estudiante pensar, pensarse y (re)pensarse como sujeto, y al mismo tiempo (re)construir esta complejidad.

Palabras clave: identidad, Trabajo Social, complejidad, (auto)biografía.

\begin{abstract}
In this paper we discuss the conceptual and methodological principles underlying the course 'Role and Identity' of the Degree in Social Work at the University of Barcelona. The main purpose of this paper is to generate a space for reflection of the concepts approach underlying the pedagogical and theoretical approach of the subject, which may be of interest to both Social Work students and professionals. For this reason, this paper takes the shape of an essay, to facilitate the reasoning and the theoretical-reflective explanation derived from the experiences, knowledge and learning of the teachers who have been part of this course.

This theoretical review of the course is tackled from two dimensions: the ontological dimension of the content of the teaching plan and the epistemological dimension of the educational methodology. In this sense, we reflect the need of Social Work students to acknowledge the underlying complexity required to understand their personal and professional reality and situates the (auto) biographical narrative as the strategy that allows students to think and (re) think themselves as subjects, and at the same time (re) construct this complexity.
\end{abstract}

Keywords: identity, Social Work, complexity, (auto) biography.

Sangrà, T.; López, J. A. y lannitelli, S. (2022). Conocimiento y aprendizaje desde la asignatura de Rol e Identidad del Trabajo Social. Itinerarios de Trabajo Social, 2, 17-25.

https://doi.org/10.1344/its.i2.36354
ACEPTADO: 14/10/2021

PUBLICADO: 21/01/2022

${ }^{123}$ Unitat de Formació i Recerca de Treball Social. Universitat de Barcelona.

$\square$ Toni Sangrà Boladeres. Unitat de Formació i Recerca de Treball Social. Universitat de Barcelona. tonisangra@ub.edu 


\section{Introducción}

En el marco de la adaptación curricular del Grado de Trabajo de Social de la Universidad de Barcelona durante el proceso de implementación del Plan Bolonia (2007-2010), entre otras asignaturas, se propuso el diseño de una asignatura obligatoria de cuarto curso llamada Rol e identidad del Trabajo Social. Esta asignatura tenia que dar un espacio al alumnado para la reflexión de los aprendizajes y conocimientos incorporados a lo largo del Grado y de su experiencia vivida.

De acuerdo con el requerimiento de avanzar sobre nuevas metodologías que se había impulsado desde el Espacio Europeo de Educación Superior, el contenido y el diseño pedagógico de esta asignatura en el nuevo Grado se impulsó y se desarrolló por un equipo de profesorado, experto y con experiencia, que asumía el reto de plantear nuevas estrategias educativas en la enseñanza del Trabajo Social.

Uno de los objetivos de la asignatura es llevar al/la estudiante a un proceso reflexivo y pensante. Ahora bien, ante esta cuestión, se nos planteaba la pregunta ¿Qué significa pensar? Por un lado, veíamos que hay una manera lógica de pensar, pensamiento deductivo: sumar, restar... Que es una forma de pensar propia del ser humano, y que se ha impuesto como único. En cambio, nuevos planteos pedagógicos nos dicen que nada nos totalice. Por esto, de lo que se trata es de des-totalizar lo que un aspecto genera sobre el resto. Ahora bien, como podemos pensar en términos calculatorios, también podemos pensar de otra manera. Pensar de otra manera significa cuestionar, y cuando se cuestiona, no se hacen cuentas, si se hicieran cuentas, no se cuestionaría, pero también sabemos que el cuestionamiento tiene un límite, por lo que, si abrimos el grifo del cuestionamiento, éste, debe ser a fondo, y en el fondo, no hay fondo... Se cae. $Y$ es justamente esa radicalidad que tiene la duda, lo que atemoriza. Ante este temor la mirada derridiana, propone abrir procesos de deconstrucción.

Entendiendo que deconstruir, no significa desarmar, significa, desmontar aquello que en su momento fue construido pero que invisibiliza su condición de construido y, se presenta como si NO fuese una construcción, sino que se presenta como propio de la naturaleza de las cosas.

Deconstrucción significa, sacar a la luz todo aquello que se nos presenta como "normal", como "verdad última" y nos demos cuenta de que también, tienen una historia, y esas historias, tienen que ver con historias de intereses, de tramas ocultas, que de algún modo benefician algún tipo de orden por sobre otro. De lo que se trata no es de destruir, sino de observar cómo se ha construido el conocimiento a lo largo de la historia, de manera que el/la estudiante no tome aquello que se le ofrece en el aula como una verdad única y pueda colocarse ahí donde él/ella considere, desde una posición reflexiva y radical, sabiendo que radical significa llegar a la raíz, en este caso a la raíz del conocimiento, sin temor.
Es desde esta premisa que la asignatura procura plantear nuevas estrategias en la enseñanza y en la construcción del conocimiento del Trabajo Social. Esta tarea se lleva a cabo desde la perspectiva biográfica y desde la transversalización conceptual con otra asignatura obligatoria de primer curso del Grado de Trabajo Social Ilamada Epistemología del Trabajo Social. Nuestro objetivo es que los elementos epistemológicos constituyan una parte del saber en el ejercicio profesional de un/a futuro/a trabajador/a social.

\section{Dimensión Ontológica de la asigna- tura de Rol e identidad del Trabajo Social}

Como hemos apuntado, no podemos desligar el sentido y el significado de los contenidos y actividades de la asignatura de Rol e identidad del Trabajo Social de cuarto curso de la asignatura de Epistemología del Trabajo Social de primer curso. El planteamiento ontológico sobre el que se construye el plan docente de la asignatura de Rol e identidad deriva de su conexión con los elementos de aprendizaje de la asignatura de Epistemología del Trabajo Social. Así pues, para la revisión ontológica de la asignatura de Rol e identidad recuperamos en primer lugar las bases conceptuales sobre las que se fundamenta la asignatura de Epistemología. Las y los docentes de esta asignatura de Epistemología apuntan:

Los diferentes apartados en los que se estructura la asignatura pretenden ir más allá de la interconexión entre perspectivas teóricas y la práctica profesional. El objetivo fundamental es posibilitar pensar sobre su propio pensamiento, sobre como construyen, asumen e incorporan el conocimiento y sobre las consecuencias en la práctica de sus posiciones epistemológicas y paradigmáticas. (Parra et al., 2012, p. 293)

En la asignatura de Epistemología del Trabajo Social de primer curso, se parte de tres elementos generales de aprendizaje para el/la estudiante: situar el estatus científico del Trabajo Social; que los/las estudiantes tomen conciencia de que las acciones prácticas en Trabajo Social son consecuencia de una opción epistemológica que viste un modelo teórico; y por último reflejar el contexto y la historicidad de los modelos de pensamiento sobre los que el sujeto construye el conocimiento.

Estas premisas y aprendizajes del primer curso en la asignatura de Epistemología del Trabajo Social son relevantes para comprender la orientación ontológica y pedagógica del plan docente de la asignatura de Rol e identidad. Así, en cuarto curso se recuperan los tres ejes del programa de Epistemología y se ponen en relación con los conocimientos, aprendizajes y experiencias que el/la estudiante ha adquirido a lo largo de su formación académica. Para cumplir este objetivo, en este momento de finalización del proceso de aprendizaje, se propone en la asignatura de Rol e 
identidad una estrategia reflexiva, de deconstrucción epistemológica, que permita el análisis de los conocimientos adquiridos durante el grado de Trabajo Social. A nivel conceptual, esta reflexión se delinea en las zonas fronterizas de la comprensión ontológica y axiológica del yo y la consecuente fragmentación de lo que llamamos "identidad". Estos contrapuntos ya señalan la naturaleza teórico-reflexiva y transdisciplinaria en que se orienta la asignatura de Rol e identidad. El objetivo pedagógico de la asignatura es permitir que el/la estudiante (re)conozca, a partir de su narrativa (auto)biográfica, el cómo ha incorporado el conocimiento relacionado con la construcción de los saberes en los que se identifica como trabajador/a social. En el contexto de la asignatura, reconocer significa re-conocer, es decir, es un volver a conocer de nuevo, no es solo señalar lo conocido de antemano. Reconocer es dejarse decir algo, y esto es un encuentro con uno/a mismo/a, contemplándose desde la singularidad y la experiencia subjetiva de su propia narrativa, ser en y a través del texto narrado como diría Roland Barthes (2004). En el relato narrado el/ la estudiante (se) re-conoce (en) aquellos aprendizajes y saberes que se vinculan con su yo trabajador/a social de manera reflexionada.

Desde esta perspectiva el relato del yo trabajador/a social, es configurador de "identidad", y el conocimiento de este "yo" no solo hay que comprenderlo, sino que ha de estar contextualizado en su objeto. Hannah Arendt sitúa el objeto en los procesos históricos de conocimiento que nos explican los límites del ser y sus realidades:

El cambio del «qué» al "cómo» implica que los verdaderos objetos de conocimiento ya no pueden ser cosas o movimientos eternos, sino que han de ser proceso, y que por lo tanto el objeto de la ciencia no es ya la naturaleza o el universo, sino la historia, el relato de la manera de cobrar existencia, de la naturaleza o de la vida o del universo. (Arendt, 2012, p.317)

Quienes somos nos remite a las preguntas: ¿Dónde estamos? ¿De dónde venimos?, ¿Adónde vamos?, es decir, la presentación de uno/a mismo/a como trabajador/a social no solo necesita un conocimiento del pasado, sino que también una orientación hacia el futuro. Es por ello por lo que se hace necesario la reflexión particular e histórica en torno a la práctica del Trabajo Social. Interrogar a la historia no para saber más cosas o cómo comportarse, sino para saber decir quiénes somos. Tal y como se plantea en la asignatura de Epistemología, preguntarse sobre el modo de comprender cambia la experiencia vivida y la proyección del yo trabajador/a social, abriendo así una posición reflexiva y de duda sobre el quienes somos, tanto desde lo personal como en lo colectivo. La elección de este camino requiere convivir con el error y con la pérdida de verdades absolutas, convivir con el error da la posibilidad de repensarse y cambiar, así mismo permite que emerja la diferencia.
Como señala Ordine (2013) la duda y la convivencia con el error no significa abrazar lo irracional y arbitrario. Desde una mirada compleja, la razón es un principio fundado en el cálculo y la lógica y, se convierte en racionalidad al entrar en diálogo con los datos empíricos, produciendo teorías. Pero si éstas abandonan el diálogo con lo real, se convierten en racionalizaciones ciegas a la experiencia, a una razón incapaz de incorporar la ambivalencia de lo real; Morin (2000) la denomina "ciega omnisciente", y ante esa posición plantea la posibilidad de una razón compleja, que concibe la realidad como un juego de orden-desorden-organización y entiende el sujeto simultáneamente como Sapiens-demens. De esta manera, la razón al dialogar con lo real incorpora al trabajo profesional la fe y la duda. Debemos estar advertidos que una razón compleja implica riesgos y peligros, pero también es cierto que la acción y el conocimiento no podrán emerger sin un cierto grado de riesgo.

De este modo, abstenerse del enfoque del cientificismo utilitarista (que no científico) predominante en la enseñanza y en las tendencias tecnológicas del Trabajo Social, y poder pensar sobre la complejidad cuestiona los prejuicios e intereses materialistas sobre los que se construye el conocimiento. En esta sociedad utilitarista, la tarea consiste en salirse de la autoreferencialidad terapéutica que caracteriza el espíritu del productivismo y del materialismo postmoderno y contemplarse en la theoría. Interpretamos la theoría en el sentido original de la palabra griega, que remite a un imbuirse de la actividad, a una participación o contemplación activa del proceso de conocer(se), que dirían Foucault (1994) o Han (2015); en definitiva, al amor (Eros) por el conocer y el saber que nos conecta con el deseo y el placer desinteresado del aprender humanista (Ordine, 2013). Cuando otorgamos primacía a la teoría, subscribimos el pensamiento no finalista que nos permite iniciar camino, pero nunca la llegada, la teoría nos abre puertas, pero nunca las cierra, la teoría nos plantea incertezas para reflexionar, nunca soluciones. El camino señala una dirección, no señala un objetivo, es decir no señala un camino prediseñado que nos lleva al lugar donde deseamos llegar. La teoría es una dirección a un territorio abierto todavía por completar. Así la teoría tiene razón de ser, en tanto esté al servicio de la actividad mental del sujeto.

Estamos, por tanto, en un debate sobre el contenido intelectual y teórico de comprensión epistemológica sobre la práctica (científica) del Trabajo Social, es decir, sobre el pensar lo que el sujeto hace o pretende hacer con el otro. Esta "exigencia teórica", que también apunta Zamanillo (2018), facilita herramientas al sujeto-estudiante que aspira a apropiarse de su propia práctica desde una posición reflexiva y crítica. Esto plantea la necesidad de desarrollar análisis teórico-prácticos sobre la complejidad de nuestros contextos, caracterizados por la continua transformación e incertidumbre, $y$ determinados por discontinuidades $y$ cruces epistémicos en los que el sujeto define su naturaleza y (re)construye constantemente sus subjetividades. 
A través de estos procesos, el sujeto define su identidad en un momento dado y configura su yo en relación con el otro. Concretamente, en nuestro mundo occidentalizado e hiper-tecnológico, la configuración del yo se da desde la fragmentación y la diferenciación con el otro, y los componentes que definen las identidades son inestables e imprevisibles. El nomadismo de las subjetividades contemporáneas define también a un sujeto ecléctico, en constante transformación y obligado a elegir constantemente múltiples opciones. La subjetividad funciona aquí como reguladora de este dinamismo que supone una negociación constante con la realidad social y con el otro dando lugar a un sujeto intersubjetivo.

En este punto, se nos hace necesario hacer un alto en el camino, para poder dirimir lo que se plantea ante el enunciado de sujeto. La noción de sujeto es una noción que llama a la controversia, ya que por sí es paradoxal, es una noción que nos remite a lo evidente y al tiempo nos remite a lo no evidente. Es evidente, en tanto podemos nombrarlo, en casi todos los idiomas existe una primera persona del singular para señalar el "Yo". Pero también es cierto, que son muchos los tratados filosóficos que hablan del sujeto como sinónimo de alma, como lo no evidente. Vivimos esta escisión en función de un paradigma profundamente enquistado en nuestra sociedad, paradigma que ya señaló Descartes, cuando planteaba que había dos mundos: uno que fundamental al conocimiento objetivo, científico (el mundo de los objetos) y el otro, un mundo intuitivo (el mundo de los sujetos). El S. XX, fue el siglo de la invasión de la ciencia clásica en las ciencias humanas y sociales. Fue el siglo en el que se expulsa al sujeto de la psicología, de la historia, de la antropología, de la sociología y, se lo reemplaza por estímulos-respuestas, es el siglo de los determinismos sociales. El estructuralismo francés declaró la "muerte" del sujeto. En la actualidad, vuelve a ser tema de debate y de reflexión, el sujeto "revive", vuelve a aparecer como realidad y categoría de análisis en las ciencias sociales. El pensamiento sociológico, antropológico... han comenzado a reflexionar en torno a la construcción de una teoría del sujeto en sociedad que otorga basamento a una consideración del actor social liberado de todo sesgo empirista o funcionalista.

Estos procesos de reflexión científica también envuelven el Trabajo Social, y la comprensión de los marcos históricos y epistemológicos que lo identifican son una estrategia de acción y aprendizaje para la construcción de las subjetividades e identidades del sujeto-estudiante. Por ejemplo, autores como Hill (1992) apuntan sobre el papel del Trabajo Social como una práctica significante y de producción simbólica en los contextos de intervención y sus efectos en las identidades del sujeto. Precisamente, el significado del concepto de "identidad" está relacionado con la producción de sentido y la subjetividad, y se confunde a menudo con una identificación del individuo más corporativa, simplista, estática y cerrada basada en unas supuestas características objetivas fruto de las influencias deterministas. Lo que llamamos "identidad" se da en las acciones significantes o representacionales, no es el sujeto quien define la identidad, sino que son los actos (de significado) los que la habilitan: "Ya que, en lo que al acto de crear significados se refiere, no hay causas de las que se pueda echar mano con certeza, sólo actos, expresiones y contextos que hay que interpretar" (Bruner, 2006, p. 126). En este sentido, en nuestros procesos de debate y reflexión, nos planteamos que puede que sea más acertado en esta asignatura denominar al concepto no como "identidad" sino como proceso identitario, que es un término que sugiere una visión más abierta, continua y que favorece el despliegue de la subjetividad.

Cuando hablamos de subjetividad, necesariamente tenemos que retomar la idea de intersubjetividad ya que supone el reconocimiento del otro. Este espacio intersubjetivo deviene el mecanismo configurador de identidades y permite al sujeto (social) vincularse a la "realidad" de su cotidianidad. La intersubjetividad opera como un esquema dialógico y de comunicación humana a través de estructuras del lenguaje que deben ser interpretadas y manifiesta la naturaleza interdependiente del sujeto. Sin embargo, nuestra sociedad líquida y comercial proyecta a un sujeto desvinculado y autónomo en el que se ignora la mirada y el vinculo del otro. Para el desarrollo de esta individualización se prioriza la autorealización y la interioridad del individuo a través de procesos de autocontrol y gestión emocional que no tienen en cuenta el papel de los demás en la conformación de nuestras identidades. Estas miradas, acomodadas en lógicas gerenciales y mercantilistas, diluyen las explicaciones colectivas y contextuales de los problemas sociales. En estas posiciones se corre el riesgo de situar al/la profesional en una concepción de su práctica puramente procedimental centrada en la gestión asistencial y emocional (Dustin, 2007).

Es por ello, que los ejes temáticos de la asignatura se configuran priorizando aquellos modelos teórico-filosóficos del Trabajo Social que tienen una base interpretativa y semiótica, como son las teorías hermenéuticas, postestructuralistas y de la complejidad. Todas ellas conectadas por el enfoque constructivista (constructive approach) que como apuntan Parton \& O’Byrne (2000) contribuyen al Trabajo Social afirmativo y reflexivo, centrado en el diálogo, la escucha y la relación con el otro, y en el que la narrativa y la poética (poesis) tienen un papel central: "The focus is narrative and different stories, and as result social work at times may take on the guise of persuasive fiction or poetry" (p. 186)

El parámetro ficcional y poético está presente en la narrativa, y es relevante en este proceso interpretativo ya que es el lugar del pensar, y su forma es la palabra que reflexiona e impulsa al sujeto-estudiante a re-construir su propio sistema narrativo basado en la conjunción de tres elementos básicos: la memoria, la metáfora y el lenguaje (Olney, 1991). La interrelación de estos elementos, que forman parte del 
mundo interpretativo de la hermenéutica, son rasgos característicos que dan significado a aquello en lo que se reconoce el sujeto, y se corresponden con la transfiguración de lo simbólico que contiene la escritura (auto)biográfica.

La narrativa (auto)biográfica recupera los significados y el valor de los sucesos y los acontecimientos experimentados. La reflexión de estos procesos de intercambio e intersubjetividad integran el yo en el mundo, creando algo nuevo y singular a partir del ejercicio de su memoria. Esto se plantea como un acto interpretativo en el que, como diría Gadamer (1993), el sujeto en un acto de (re)conocimiento transforma y rehace sus materiales interiores (recuerdos y emociones) y el yo se (con)forma en la conciencia a través de la interacción con el medio.

\section{Dimensión Educativa y Metodológica de la asignatura de Rol e identidad}

\subsection{Epistemología pedagógica}

El marco conceptual y epistemológico de la tarea docente y educativa que define la asignatura de Rol e identidad del Trabajo Social se orienta en torno a las conclusiones elaboradas por el informe de la UNESCO de la Comisión Internacional sobre la Educación para el Siglo XXI presidida por Jaques Delors. Este informe que lleva por título "La educación encierra un tesoro", fue publicado en 1996. Entre las principales conclusiones se identificó cuatro pilares en el proceso de aprendizaje: aprender a conocer, aprender a hacer, aprender a ser y aprender a convivir. Según el informe es necesario entender la educación como un todo (Delors, 1996) y, al igual que Edgar Morin, quien lideró el informe "Los siete saberes necesarios para la educación del futuro", también publicado por la UNESCO el 1999, se interpela a los/las docentes, académicos/as y profesionales como responsables de una reforma del pensamiento adecuada a los nuevos retos del siglo XXI.

Por tanto, el posicionamiento docente y epistémico viene determinado por los escenarios educativos, sociales, políticos y culturales ligados al siglo XXI (Beck, 1998; Morin, 2000). Estas realidades sitúan el reto educativo en la formación de los sujetos desde la complejidad, colocando los procesos reflexivos e interpretativos como base para el aprendizaje. Morin et al. (2013) razonan en torno a estos desafíos y el papel que debe tener la educación del futuro en relación con la deconstrucción-reorganización de los saberes y conocimientos, es decir, con la reflexión y comprensión de por qué se sabe, lo que se sabe. Nuestra propuesta se enmarca, pues, en esta idea reformista que plantea Morin en la que se busca profundizar en aquellas metodologías de aprendizaje que favorecen la adquisición de conocimientos vinculados a un pensamiento que une la complejidad del saber y el contexto. Unos conocimientos que se fundamentan en la reflexión interpretativa, la comunicación y las relaciones intersubjetivas.
Nos situamos en un territorio epistemológico y pedagógico complejo que busca alterar los formatos clásicos de transmisión del conocimiento. Esta dimensión más centrada en la proximidad hacia el/la estudiante y sus necesidades desarrolla acciones docentes que descentran la orientación disciplinaria, lineal y progresiva de la enseñanza tradicional. Es decir, en la organización y la experiencia docente ya no se trata de reconocer una serie de verdades universales o de argumentar un razonamiento práctico (enraizado al conocimiento arbóreo de ciencia moderna); sino que trata de asumir las potencialidades existentes en los sujetos-estudiantes a través, en este caso, de la reflexión biográfica para que estos puedan orientarse en el tránsito de sus "verdades" e intuiciones morales. Entendemos que los saberes se hallan inmovilizados por lo acordado y establecido en la ciencia moderna de la tradición (Ilustración), y estas premisas (de como conocer y que es saber) se encuentran fijadas en la formación (académica) y el aprendizaje del/la estudiante. Existe en cierto modo, como apunta Nuccio Ordine (2013), un interés socioeconómico que determina la producción de conocimiento de tipo utilitarista, que busca ser una oferta dentro de la cadena de las demandas. Siguiendo la idea de Ordine, tenemos la impresión de que la academia tiende a burocratizarse, y que las fuerzas creativas comienzan a flaquear. Así, aspiramos a una epistemología educativa crítica y autónoma, que pueda superar la función adaptativa y reactiva de los modelos educativos deterministas y que aspire a un desarrollo multidimensional del sujeto. No en vano, también en el intento de explicarnos (en este texto y en nuestra actividad docente) seguramente lo transformamos en un cerrajón que inmoviliza el saber. Precisamente, partimos de este reconocimiento autocritico para abrirnos a experimentar con narrativas alternativas a las asociadas a la cultura moderna y a las ingenierías productivistas, con el objetivo para dar respuesta, o al menos intentarlo, a las principales problemáticas de nuestra época. En este "laboratorio" intentamos que el conocimiento sea compartido y construido entre estudiante y docente. La experiencia de aprendizaje (también por parte de el/la docente) permite estimular en los/las estudiantes la participación y la acción crítica (como sujetos proactivos), al tiempo que potenciar una actitud más interpretativa que conlleva un aprender desde el crear y el recibir (Gadamer, 1993). En este sentido Gadamer, plantea que el horizonte se desplaza al paso de quien se mueve. A partir de aquí, se entiende que el significado no preexiste, sino que es creado en el proceso de interacción, así la interpretación no hace referencia sólo a la interacción, sino también a lo que se ha dicho en la interacción.

De este modo se plantea una experiencia dialógica y comprometida de aprendizaje que busca replantear la enseñanza más materialista, utilitarista y unidimensional (Ordine, 2013). Es decir, se apuesta por dinámicas educativas complejas y dialógicas que aspiren a que el sujeto tome conciencia de un conocimiento paradigmático basado en la práctica y la reflexión. 
Derivado de esto, si favorecemos esta actividad del conocer estamos dando respuesta a los retos que nos presenta un contexto educativo y sociopolítico que ha restringido la forma de conocer en base a resultados y contenidos estandarizados. La formación en la complejidad, por lo tanto, supone impulsar un pensamiento ligado a la práctica y los problemas derivados de la experiencia humana; problemas que las y los futuros profesionales del Trabajo Social deberán afrontar y comprender desde una inteligencia multidimensional y transdisciplinar (Morin, 2000).

A nuestro entender la educación no es sinónimo de acumulación de conocimiento, sino que se refiere a la emoción y al cultivo de un espíritu curioso por el saber. Si la apuesta de la universidad pasa por el ejercicio de la ciudadanía de todos y cada uno de sus actores (docentes, estudiantes, personal administrativo... ) por el comportamiento ético, responsable, estético, creativo, debemos recuperar la relación entre educación y la reflexividad de los valores críticos, ocuparnos y preocuparnos por los sujetos y de las razones que conforman las biografías.

A pesar de que la neutralidad economicista y racionalista impregna cada vez más las estructuras educativas, también es cierto que el aprendizaje tiene lugar en el ámbito privado, paradójicamente cuando hablamos de aprendizaje nos estamos refiriendo a un fenómeno social, como seres gregarios aprendemos de otros, con otros. Todos somos producto de conversaciones anteriores, todos somos una combinación de la conversación de otras personas, muchos de cuyos valores retenemos.

Evidentemente después de cinco siglos de certezas racionales y un contexto globalizado que afecta a las formas de relacionarnos, si optamos por la educación desde las biografías, necesitamos un cambio profundo del sistema educativo centrado en una educación ligada a los valores críticos y democráticos. Cuando hablamos de sistema, estamos hablando de organización tal como plantea Morin (2000), estamos hablando de sujetos vivos, por lo que el cambio debe empezar por todos y cada uno de los participantes en el escenario educativo. El cambio no puede ser sólo metodológico, sino que también requiere de un cambio en la operatoria universitaria, creando condiciones materiales y potencialidades pedagógicas que permitan la construcción de nuevas formas de convivencia, para que el conocimiento deje de ser un objeto externo y neutral a las desigualdades y a la precarización del sujeto.

\subsection{Narrativa y (auto)biografía}

Para el desarrollo del plan conceptual y temático de la asignatura de Rol e identidad se apuesta por propuestas educativas basadas en metodologías narrativas con el objetivo que el/la estudiante identifique el lugar que ocupan los marcos referenciales de sus aprendizajes y experiencias relacionadas con la construcción de su yo trabajador/a social.
El planteamiento metodológico y la actividad narrativa que se propone en el marco de la asignatura se concreta en la elaboración de una narrativa (auto) biográfica reflexionada de acuerdo con los marcos y contenidos teóricos del plan docente, y que son trabajados en espacios de seminario y reflexión durante el curso.

En concreto, se propone la elaboración de una narrativa autobiográfica en la que el/la estudiante pueda compenetrarse y reconocer como ha ido incorporando y transformando el conocimiento, para después poder interpretar aquellos marcos referenciales que afectan su impregnación en la subjetividad y el efecto en la construcción de sus identidades. En un segundo momento, en el ejercicio de interpretación narrativa se incorporan en la narrativa biográfica los conceptos teórico-reflexivos clave desarrollados y trabajados durante el curso.

Para contextualizar brevemente la metodología (auto)biográfica, apuntamos en primer lugar que en las ciencias sociales y humanas la metodología biográfica es una estrategia (de investigación y acción) cualitativa y etnográfica: "En el método autobiográfico, el sujeto es el actor y protagonista del relato, por este motivo se requiere un enfoque ideográfico (centrado en el individuo) y cualitativo, que implica la característica de ser inductivo" (Sanz, 2005, p. 106). Los sujetos no se reducen a variables, sino que son considerados como un todo dentro de un contexto ecológico, social, cultural, histórico. Esta estrategia conecta con el enfoque humanístico, interpretativo y fenomenológico en el que se apoya la mirada epistemológica y ontológica de la asignatura. Así mismo, tanto la asignatura como la metodología (auto)biográfica abraza diferentes campos de conocimiento que van desde la sociología y la antropología hasta el arte. Esta transdisciplinariedad nos plantea el reto de entender las narraciones autobiográficas más allá de una acción didáctica.

Históricamente las formas autoreferenciales como diarios, historias de vida, biografías, cartas, etc., centradas en la presentación del “yo", toman cuerpo en el proceso de modernización de la sociedad con la constitución del primer capitalismo fordista y el nacimiento del espíritu burgués. En líneas generales, la escritura autobiográfica como referencial narrativo de identidad la inician autores literarios como Goethe o Rousseau en el siglo XVIII, que sientan las bases de la novela moderna, y que mediante la narrativa refunden una nueva vida. En el siglo XIX, también los elementos autobiográficos se plasman en los relatos sobre la vida social de autores enmarcados en el romanticismo y el realismo como Baudelaire, Dickens, Balzac o Zola. Por otro lado, la autoreferencialidad existencial también deviene para artistas y escritores del siglo XX como un modo de resistirse a un orden sociocultural, que paradójicamente promueve el sentido de individualidad, haciendo saltar el sí mismo a la zona del espacio público, y por lo tanto otorgando ya un lugar al otro. 
En el abanico de literaturas del yo, la forma autobiográfica es la más comúnmente conocida y aceptada. Este género de la memoria lo formaliza Philippe Lejeune en el texto "El Pacto autobiográfico" en el que, tal y como sugiere en el título, plantea un "pacto" para la lectura de la autobiografía en la que se asume la referencialidad real del sujeto biografiado; Lejeune afirma que la autobiografía es un "relato retrospectivo en prosa que una persona real hace de su propia existencia, poniendo énfasis en su vida individual y, en particular, en la historia de su personalidad" (Lejeune, 1994, p.50).

En la obra de Lejeune se da por buena la mismidad del autor, narrador y protagonista, y entre este triangulo identitario se "pacta" con el lector una verdad. Sin embargo, este acuerdo no es tan simple como apunta Paul De Man (2007), ya que por un lado en "el momento autobiográfico ocurre como un alienamiento entre los dos sujetos involucrados en el proceso de la lectura en el que se determinan el uno al otro mediante una mutua sustitución reflexiva" (De Man, 2007, p.149); y por el otro lado, la fricción narrativa de las diferentes figuras identitarias (autor-narrador-protagonista) "des-figuran" la linealidad del contorno que el sujeto dibuja sobre si mismo; es decir, en la acción de narrarse se manifiesta la existencia del sujeto al plasmarse en una estructura de lenguaje (grafía) y al diseminarse este entre lo simbólico y lo vivido (auto y bio).

La autobiografía más allá de las formas literarias que adopta como las memorias o historias de vida, las novelas personales, los diarios íntimos, epistolarios, notas en cuadernos de trabajos o ensayos sobre uno mismo, etc., sugiere, en términos de Arfuch (2010), un espacio de reflexión filosófico, social y político:

La necesidad de la autobiografía adquiere así relevancia filosófica: no solamente explora los límites de la afectividad abriendo paso a un nuevo género, entre las tendencias literarias de su época; no sólo expresa el sentimiento de acoso y de defensa frente a la intrusión de lo íntimo por lo social -en la interpretación de Arendt-, sino que introduce la convicción íntima y la intuición del yo como criterios de validez de la razón. (Arfuch, 2010, p. 44)

En esta argumentación, tal y como hemos apuntado anteriormente, no podemos dejar de plantearnos la cuestión ontológica de la identidad del “yo" (en tanto adscripción lingüística que define al sujeto enunciante) y la cuestión epistemológica de cómo lo conocemos. Como nos recuerda Félix Guattari (1990) el sujeto no es evidente, y la duda siempre recae en que aquello que existe puede ser distinto de aquello que podemos conocer.

De modo resumido, la comprensión que hacemos de la metafísica interior del yo responde a dos líneas histórico-sociales: por un lado, al referente divino o espiritual que la fe da al sujeto, dando sentido a su existencia, y que ha servido y sirve para sostener el concepto de la dignidad y justicia humana; y por el otro, a la estructuración cientificista, derivada de los modelos socio-económicos dominantes, que articula prácticas de subjetivación que distorsiona la mismidad en base a lógicas gerenciales y de capital emocional (Illouz, 2007).

Sin querer entrar ahora en un debate controvertido y fuera de lugar en este momento, apuntamos que desde la modernidad occidental se ha privilegiado la idea de un yo interior, en el que el sujeto atesora en la profundidad de su ser sus pensamientos e ideas más genuinas, dejando en el espacio exterior el mundo sensitivo (sujeto-objeto). Como señala Charles Taylor (2006) el constructo actual del yo, que históricamente vincula al sujeto occidental a un orden moral, se ha ido gestando desde el siglo XVIII hasta la cultura individualista de la modernidad que ha desplazado su comprensión hacia el mundo psíquico e intrapersonal. Así, compartiendo con Taylor, la imposibilidad de desvincularse del todo de estos marcos referenciales históricos y contextuales, es precisamente la reflexión y la interrogación que el sujeto puede proyectar sobre estos lo que le define: "Lo que soy como un yo, mi identidad, está esencialmente definido por la manera en que las cosas son significativas para mí" (Taylor, 2006, p. 61). En este ejercicio reflexivo el yo aparece como una especie de material histórico compartido con el otro en un mundo subjetivo y con capacidad de reconfigurarse: "Uno es un yo sólo entre otros yos. El yo jamás se describe sin referencia a quienes lo rodean" (Taylor, 2006, p. 62). En definitiva, estamos ante una abstracción de organización compleja, temporal e intersubjetiva que adquiere forma narrativa sin entidad fija: "La comprensión que tengo de mí mismo necesariamente tiene una profundidad temporal e incorpora la narrativa." (Taylor, 2006, p. 83). De este modo, la conciencia, el solipsismo y el ego son primera persona de narración ya narrada, producto de una historia contada o por contar.

El conocimiento de la realidad desde la narrativa (auto)biográfica se inscribe en nuevos espacios de reelaboración subjetiva de este conocimiento, es decir, produce conocimiento en un contexto de realidad al mismo tiempo que subjetiva esa realidad para construir el yo. En este caso, por lo tanto, se descarta como un proceso psy de autoconocimiento: "El interés de la autobiografía, entonces, no reside en que revela autoconocimiento fiable -no lo hace- sino en que demuestra de manera sorprendente la imposibilidad de cierre o de totalización" (De Man, 2007, p. 149-150). Desde la óptica constructivista, que contiene la narrativa como construcción biográfica de la existencia, la realidad se constituye en la imagen, en ese tropo que formaliza (da Forma) a la vida. Es decir, la imagen de uno mismo es un producto de las narrativas propias, y como apunta Ricoeur son producto del relato de diferentes voces narrativas (máscaras) culturales y contextuales: "En lugar de un yo (moi) enamorado de sí mismo, nace un sí (soi) instruido por los símbolos culturales" (Ricoeur, 2006, p. 22). 


\section{Reflexiones finales}

Tal y como hemos ido apuntando en los apartados anteriores, los tres ejes expuestos, lo ontológico, lo pedagógico y lo narrativo, que configuran la idiosincrasia de la asignatura, confluyen en el/la estudiante que es el/la protagonista vivencial de las cuestiones planteadas. En nuestra propuesta, centrada en el pensamiento reflexivo y complejo, se revaloriza el papel del sujeto-estudiante en la construcción del conocimiento. Es decir, más allá de decir algo es así o está así, el sujeto-estudiante puede interrogarse sobre los supuestos que generan acciones morales a priori inmutables, y a partir de aquellos contextos en los que cobran sentido cuestionar las verdades que los legislan.

En el camino hacia el conocimiento, la búsqueda de "la verdad" es un imposible, avanzamos hacia el conocimiento a través del diálogo con el otro. Es por ello, que es en y por el lenguaje que el ser humano se construye como sujeto. Los sujetos son designados por otros sujetos. La palabra designa desde antes del nacimiento, no sólo el estatuto de sujeto, sino la llegada al mundo de un ser biológico. Así, plantearse el giro narrativo y biográfico es una oportunidad para trascender en las formas de saber y de conocer más allá de la conocidas posiciones realistas y positivistas. En nuestro espacio disciplinario del Trabajo Social, este giro epistemológico puede sugerir un cambio en el eje de la comprensión del conocimiento de la acción social.

Por ello, conectamos este trabajo con el contexto actual contemporáneo, caracterizado por su tendencia a la recuperación historicista de la memoria y la identidad desde múltiples enfoques. Esto es así, por ejemplo, en la restauración de las memorias históricas o el interés cultural y patrimonial en lo real mitologizado, o también en el marketing masivo de la nostalgia. En todo caso, a pesar del fulgor por lo autobiográfico y las prácticas de la memoria, nuestro trabajo aquí no trata de proyectar una dependencia al orden hegemónico del espacio de representación; sino de desplegar un desarrollo de estrategias para la reflexión sociopolítica del sujeto y su efecto transformativo en la subjetividad.

Nuestra propuesta pasa por el hecho de que la educación asuma una función habilitadora, entendiendo que la educación es un proceso de construcción, donde el papel activo lo desempeña el/la estudiante, de manera que le permita el cultivo de todas sus capacidades y que le dote de las herramientas intelectuales y sensibles para el desempeño de sus funciones en una sociedad compleja, dinámica y cambiante. En la asignatura de rol e identidad, deseamos que el sujeto-estudiante desarrolle sus propias estrategias para el conocimiento, facilitando que incorpore también estrategias de intervención, por lo que al sujeto-autobiografiado se le hace imprescindible hacer emerger los aspectos ético-estéticos presentes en el acto de crear. En este contexto, arte y ciencia son un dúo indisoluble, al igual que la ética y la estética, y juntamente con las nuevas formas de hacer, vinculadas a los procesos sociales y culturales característicos de nuestro mundo contemporáneo, que desdibujan estas dicotomías, da lugar a poder pensar también el Trabajo Social como creación, y revalorizarlo en su potencialidad emancipadora y democrática.

\section{Referencias bibliográficas}

Arendt, H. (2012). La condición humana. ( $7^{\mathrm{a}}$ ed.). Paidós. Arfuch, L. (2010). El espacio biográfico. Dilemas de la subjetividad contemporánea. ( $1^{\mathrm{a}}$ ed.). Fondo de Cultura Económica.

Barthes, R. (2004). Roland Barthes por Roland Barthes. ( $1^{\text {a }}$ ed.). Paidós Contextos.

Beck, U. (1998). La sociedad del riesgo. Hacia una nueva modernidad. ( $1^{\mathrm{a}}$ ed.). Paidós.

Bruner, J. (2006). Actos de significado: más allá de la revolución cognitiva. ( $1^{\mathrm{a}}$ ed.). Alianza.

De Man, P. (2007). La autobiografía como des-figuración. En: La retórica del Romanticismo. Akal.

Declaración conjunta de los ministros Europeos de Educación reunidos en Bolonia el 19 de junio de 1999 (Declaración de Bolonia). http://universidades. universia.es/fuentes-info/documentos/bolonia.htm

Delors, J. (1996). Los cuatro pilares de la educación. En: La educación encierra un tesoro. Informe a la UNESCO de la Comisión Internacional sobre educación para el siglo XXI. Santillana/UNESCO.

Dustin, D. (2007). The McDonaldization of Social Work. $\left(1^{\mathrm{a}}\right.$ ed.). Ashgate.

Foucault, M. (1994). Hermenéutica del sujeto. ( $1^{\mathrm{a}}$ ed.). La Piqueta Endymión.

Gadamer, H. (1993). Verdad y Método. ( $3^{\mathrm{a}}$ ed.). Sígueme. Guattari, F. (1990). Las tres ecologías. (2 ${ }^{\mathrm{a}}$ ed.). Pre-Textos. Han, B. (2015). La sociedad del cansancio. (8a ed.). Herder Hill, R. (1992). Nuevos paradigmas en Trabajo Social. Lo social natural. ( $\left.1^{\mathrm{a} e d}\right)$. Siglo XXI

Illouz, E. (2007). Intimidades congeladas. ( $1^{\mathrm{a}}$ ed.). Katz Editores. Lejeune, P. (1994). El pacto autobiográfico y otros estudios. ( $1^{\text {a }}$ ed.). Megazul-Endymion

Morin, E. (2000). Lamente bien ordenada. ( $1^{\mathrm{a}}$ ed.). Seix Barral. Morin, E., Roger, E., Domingo, R., y (2013). Educar en la era planetaria. ( $1^{\mathrm{a}}$ ed.). Gedisa.

Parton N., y O’Byrne, P. (2000). Constructive Social Work. Towards a new practice. ( $1^{\mathrm{a}}$ ed.). Palgrave.

Olney, J. (1991). Algunas versiones de la memoria/Algunas versiones del bios: la ontología de la autobiografía. Anthropos, 29, pp. 33-47.

Ordine, N. (2013). La utilitat de l'inútil. (1 ${ }^{\mathrm{a}}$ ed.). Quaderns Crema.

Parra, B., Iannitelli, S., y López, T. (2012). Reflexividad y epistemología en la enseñanza del Trabajo Social. En: Emma de Sobremonte de Mendicuti (Ed.). Epistemología, teoría y modelos de intervención en Trabajo Social: Reflexión sobre la construcción disciplinar en España (p. 293-299). Deusto Digital.

Ricoeur, P. (2006). La vida: un relato en busca de narrador. Ágora, 25(2), 9-22. 
Sanz, A. (2005). El método autobiográfico en investigación social: potencialidades y limitaciones de las fuentes orales y los documentos personales. Aclepsio Vol. LVII, 1, 99-115.

Taylor, C. (2006). Fuentes del yo. La construcción de la identidad moderna. ( $1^{\mathrm{a}}$ ed.). Paidós.

Zamanillo, T. (2018). Epistemología del Trabajo Social. De la evidencia empírica a la exigencia teórica. ( $1^{\mathrm{a}}$ ed.). Ediciones Complutense. 
